\title{
Selection of the most suitable decomposition filter for the measurement of fluctuating harmonics
}

\author{
Jorge Bruna*, Julio J. Melero ${ }^{\dagger}$ \\ CIRCE - Universidad de Zaragoza \\ C/ Mariano Esquillor 15 - 50018 Zaragoza, Spain \\ Phone: +34 976761863 \\ Email: *jbruna@fcirce.es, ${ }^{\dagger}$ melero@unizar.es
}

\begin{abstract}
The proliferation of non-linear loads in both industrial and residential distribution grids leads to undesirable non-sinusoidal and fluctuating harmonic pollution on voltage and current waveforms. New analysis tools, such as wavelets, are being used to overcome the problems posed by the use of the Fourier Transform when analyzing complex waveforms. Nevertheless, the selection of the wavelet basis must be done carefully to minimize spectral leakage due to the non-exact frequency discrimination. In this context, this paper proposes an objective method for comparing different wavelet families for the measurement of harmonic contents. This methodology is applicable for determining the best filter among the 53 preselected structures according to the following requirements: frequency selectivity, computational complexity, convolution results and observed spectral leakage. With all these considerations, the Butterworth IIR filter of order 29 was found to be the best wavelet decomposition structure to achieve an effective harmonic analysis up to the $\mathbf{5 0}^{\text {th }}$ order.
\end{abstract}

Index Terms-Convolution, FIR digital filters, Harmonic analysis, IIR digital filters, Wavelet packets, Wavelet transforms.

\section{INTRODUCTION}

$\mathbf{T}$ HE recent development of distributed generation systems brings the integration of a large number of small-scale generators into distribution grids. The paradigm shift from centralized to distributed systems poses new challenges to energy management and control. As a result, small-scale generators are increasingly incorporating power-electronic based converters. These converters require distorted currents from the electrical system resulting in non-linear patterns of energy consumption [1]. In this regard, several power-quality-related standards, such as IEC 61000-3-7 [2] and IEC 61000-3-15 [3], have been developed to measure and limit harmonic emissions for the connection of non linear loads. The use of these relatively new standards in conjunction with the traditional ones, IEC 61000-4-7 [4] and IEEE 519 [5], allows the evaluation of harmonic emissions in distributed systems, assuming sinusoidal and steady-state nature. However, these conditions are less likely to be found in practice. As an example, Pulse-Width Modulation (PWM) techniques, which are used today as the basis for energy conversion in distributed power sources, frequently operates under voltage disturbances like harmonics, flicker fluctuations and unbalances [6]. It is therefore highly recommended to perform harmonic measurements with methods capable of taking into account these special characteristics [7].
Harmonic measurement methods are mainly based on the Fourier transform (FT) and they are only valid under sinusoidal steady state conditions. The enhanced Fast Fourier Transform (FFT) is the most widely used method for harmonic measurement. It was developed and adopted for stationary harmonic analysis; however, it is not appropriate for fluctuating harmonic assessment due to its excessive spectral leakage and undesirable picket fence effects [8]. Short-Term Fourier Transform (STFT) theory can avoid these problems by using window functions, but the flexibility of harmonic detection is reduced [9], [10]. Other techniques like the Wavelet Transform (WT) can also be applied for harmonic analysis. This theory is currently helping researchers to develop better algorithms in terms of non-sinusoidal and fluctuating measurements [11]. Moreover, recent studies confirm that the WT is also valid for the determination of other advanced power quality (PQ) parameters such as events, unbalance or flicker [12]-[19].

Wavelets are being applied to some different aspects of electrical engineering and in particular to harmonic measurement. Broadly speaking, mother wavelets are irregular, asymmetric and with a certain number of vanishing moments (directly linked with energy concentration after decomposition). This, in contrast with the FT functions (sine waveforms), allows WT to analyze distorted signals more accurately than FT [20]. Therefore, the adequate selection of the mother wavelet can minimize spectral leakages when decomposing the input waveform into smaller frequency bands [21].

Over the past, many important contributions have been made to the use of wavelet decomposition. Nonetheless, no explanation has been provided on the use of certain types of wavelet families on a complete harmonic analysis [22], [23].

For example, Zhu et al. [24] listed some aspects to be considered when choosing the mother wavelet family by explaining the characteristics of Daubechies mother wavelets (db1, db4, db24 and db40) applied to power harmonics. One year later, Yuang et al. [25], who cited Zhu's work [24], selected $\mathrm{db} 2$ wavelet family without mentioning the technical justification for that choice. Other works used not only classic Finite Impulse Response (FIR) filters but also other Infinite Impulse Response (IIR) structures to decompose the signal under a Wavelet Packet Transform (WPT) approach to enhance frequency discrimination performance [26]-[28]. It is clear that frequency discrimination concerns authors in order to improve the existent electrical algorithms. For instance, [29], 
[30] used different structures on their harmonic analysis, such as Daubechies, Vaidyanathan, Coiflet or Johnston's filters, but both authors only compared filter's performances regarding the obtained deviations. Other authors only studied energy concentration in the time-frequency plane to detect electrical disturbances through the determination of adaptive thresholds [31].

As noted, there are many works related to the use of wavelets but there is not an objective methodology to be followed on the selection of the most suitable wavelet family (or filter) on the first design stages. This paper proposes a methodology that relies on the selection of the best filter depending on its intrinsic characteristics such as frequency selectivity, computational cost and low spectral leakage, considering not only FIR filters but also IIR ones. This research is supported by the results obtained through the use of a WPTbased algorithm which allows the measurement of even and odd harmonic contents up to $63^{\text {rd }}$ order, beyond the mandatory requirements of the standards [2], [3].

The paper is structured as follows. First, a general justification of the chosen methodology is provided (Section II-A). Section II-B shows the proposed decomposition tree with a brief review of the most relevant Quadrature Mirror Filter (QMFs) characteristics. Besides, the WPT decomposition is implemented through certain convolution and nodal aggregation operators. Frequency and computational criteria are also described in Section III with some considerations prior to implementation that will guide developers in the first selection stages. In Section IV the convolution techniques are studied and the most appropriate is selected. In Section V we present the results obtained in some of the conducted tests (pure-tone and fluctuating harmonic analysis) including the most relevant wavelet filters. Finally, conclusions are presented in Section VI.

\section{DECOMPOSITION STRUCTURE}

\section{A. Principle of the decomposition scheme}

Advanced harmonic measurements can be performed with the Discrete Wavelet Transform (DWT) in a similar way that it is done with the FFT. The analyzed signal is decomposed into different frequency contents and then, individual harmonics are computed. In the DWT, the initial waveform is just passed through a wavelet filter by means of the convolution operator providing a certain division of the frequency domain.

In this paper, the Wavelet Packet Decomposition (WPD) has been used to obtain the harmonic spectrum of the input signals. WPD can be described as a Multiresolution Analysis (MRA) method where the output of the current level is downsampled and fed into the next level recursively. The outputs $y$ of each level are extracted by the wavelet filters through the convolution process of the input signal $f$, and they are then decimated by a factor of 2 [23].

$$
\begin{aligned}
& y_{H P}[k]=\sum_{n} f[k] g[2 k-n] \\
& y_{L P}[k]=\sum_{n} f[k] h[2 k-n]
\end{aligned}
$$

All high-pass filtered data give the detail coefficients whereas the low-pass filtered data are identified as approximation coefficients, having both half of the original input data due to downsampling process. This decomposition procedure halves the time resolution since only half of the samples now characterize the initial content. On the contrary, this operation doubles the frequency resolution, since the frequency band of the signal spans only half of the previous frequency band, reducing the frequency uncertainty by 2 [32].

As stated by Barros et al. [33], the wavelet packet analysis is perfectly recursive; in other words, the low-pass and highpass filtering process is repeated all over the decomposition tree, from the first level to the last one. This process gives as many nodes as $2^{n}$, being $n$ the number of levels that integrate the tree. Thus, with a careful selection of sampling frequency, measuring window length, convolution technique and decomposition filter, the output nodes at the last decomposition level are able to represent the energy contained by each fundamental harmonic.

\section{B. Implementation methodology}

The MRA-based method relies on the use of decomposition structures called quadrature mirror filters. They are formed by a pair of complementary filters, one low-pass and one high-pass, which split the frequency range into two equal parts (Figure 1). QMF have been extensively used in digital signal processing (DSP) and especially in the application of WPD schemes. They are used in this paper to decompose the input signal into two frequency intervals from 0 to $\frac{\pi}{2}$ radians and from $\frac{\pi}{2}$ to $\pi$ radians. After the filtering process, half of the samples can be discarded according to the Nyquist sampling theorem since the signal now has a highest frequency of $\frac{\pi}{2}$ radians instead of $\pi$. The process of downsampling is performed by a decimation operator which subsamples the signal by a factor of 2 . This halves (shrinks) the time resolution but doubles (stretches) the frequency resolution [9] according to the Heisenberg's uncertainty principle. Additional mathematical background of the WT and WPD tools can be found in several reference publications [34], [35].

Figure 1 illustrates the frequency response of a QMF pair. It describes both low-pass and high-pass filters used in the MRA scheme (with magnitude responses forming a mirrorimage symmetric pair around $\omega=\frac{\pi}{2}$ ).

The final objective of the MRA scheme is the extraction of frequency information for each harmonic order at the last decomposition level. The coefficients that allow such calculation are obtained by a recursive algorithm developed from the binary tree composed by the selected filters (Figure 2). The algorithm is built from a decomposition tree of 7 levels and is capable of measuring up to the $63^{\text {rd }}$ harmonic order. The Root Mean Square (RMS) information can be obtained by computing the aggregation of 2 output nodes with a frequency span of $25 \mathrm{~Hz}$ [26], [36]. This gives the centered harmonic RMS with a bandwith of $\pm 25 \mathrm{~Hz}$ (3).

$$
x_{r m s}(j, p+q)=\sqrt{\frac{\sum_{k}\left(d_{j, k}^{p}\right)^{2}+\sum_{k}\left(d_{j, k}^{q}\right)^{2}}{N}}
$$


where $d$ is the wavelet coefficient, $p$ and $q$ are the combined nodes, $N$ the number of data in the measurement window, $k$ referes to index counter and $j$ is the level in which the RMS is calculated.

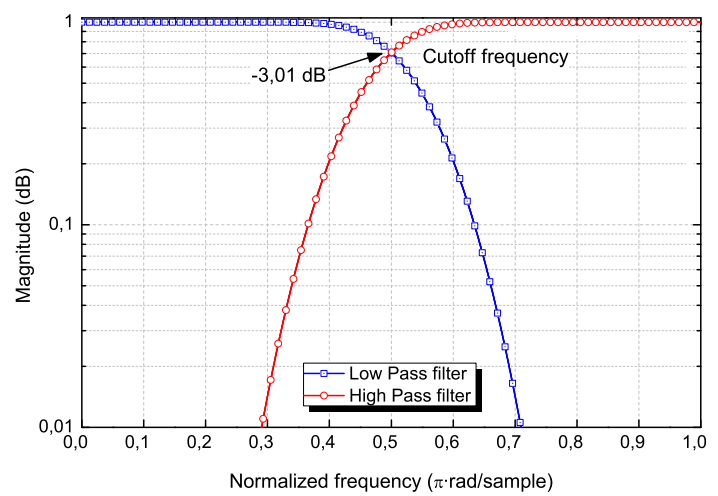

Figure 1: Quadrature Mirror Filters

This development is based on the IEC 61000-4-7 standard guidelines [4] in order to be compliant with its minimum requirements, which are: 10-cycle time interval for $50 \mathrm{~Hz}$ power systems, harmonic measurements up to $2 \mathrm{kHz}$ and use of rectangular convolution windows. Then, the sampling frequency is set to $6400 \mathrm{~S} / \mathrm{s}$ with measuring windows of $200 \mathrm{~ms}$. Table I summarizes the developed method performance at any node, including information about the number of nodes per level, samples per node, effective time resolution and bandwidth for every node at any level. As can be seen in Table I, the $7^{\text {th }}$ decomposition level has a limited bandwidth of $25 \mathrm{~Hz}$. Following the standard guidelines [4], the harmonic content must be centered; so, given the aforementioned requirements, 2-nodal-groupings of $\pm 25 \mathrm{~Hz}$ per harmonic are mandatory.

Table I: Decomposition tree summary

\begin{tabular}{ccccc}
\hline Level & Nodes & $\begin{array}{c}\text { Samples } \\
\text { per node }\end{array}$ & $\begin{array}{c}\Delta t \\
(\mathbf{m s})\end{array}$ & $\begin{array}{c}\text { Bandwidth } \\
\text { per node }(\mathbf{H z})\end{array}$ \\
\hline 1 & 2 & 640 & 0.312 & 1600 \\
2 & 4 & 320 & 0.625 & 800 \\
3 & 8 & 160 & 1.250 & 400 \\
4 & 16 & 80 & 2.500 & 200 \\
5 & 32 & 40 & 5.000 & 100 \\
6 & 64 & 20 & 10.000 & 50 \\
7 & 128 & 10 & 20.000 & 25 \\
\hline
\end{tabular}

\section{FILTER SELECTION: FREQUENCY SELECTIVITY AND COMPUTATIONAL COMPLEXITY}

The first step when performing a WT decomposition consists in selecting the mother family. Once selected, it is possible to develop the transform methodology for a particular application. In this paper we are looking for the optimal wavelet mother family (filter) for harmonic measurement. To identify the most suitable one, we have first chosen 53 filters (or wavelet mother families). The following wavelet families and filters have been selected: Haar (FIR), Daubechies

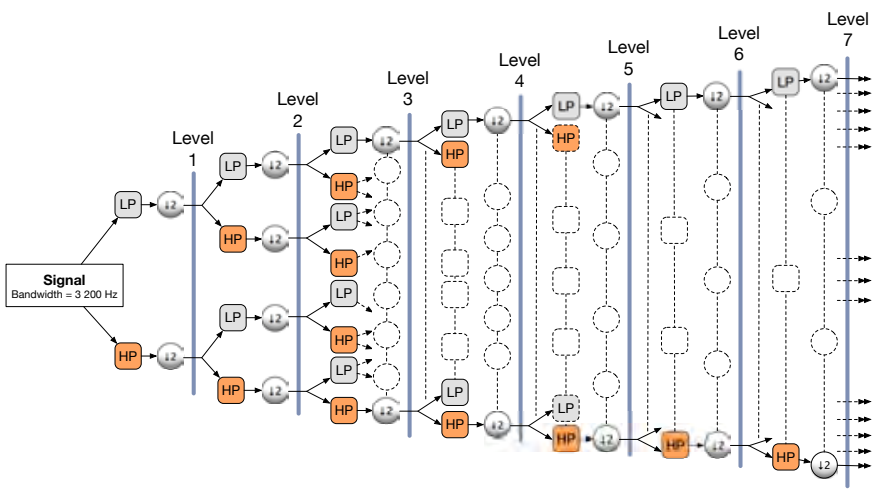

Figure 2: Full decomposition tree

(db1-db20) (FIR), Coiflets (coif1-coif5) (FIR), Symlets (sym2sym10) (FIR), Discrete Meyer (FIR), Biorthogonal (bior1.1bior1.5, bior2.2-bior2.8, bior3.1-bior3.9, bior4.4, bior5.5 and bior6.8) (FIR), Vaidyanathan (FIR), Elliptic (IIR) and Butterworth (IIR). This 53-filter list extensively covers all possible case studies in terms of type of response to impulses (FIR and IIR), symmetry, orthogonality, biorthogonality and vanishing moments.

In terms of frequency selectivity, IIR decomposition filters perform better than the traditional ones (e.g. db10, db20 or Vaidyanathan FIR filters) [26]. Complex frequency responses were obtained through the evaluation of the filter transfer functions at any given frequency $\omega(4)$.

$$
H\left(e^{j \omega}\right)=\frac{\sum_{k=0}^{M-1} b(k) e^{-j \omega k}}{\sum_{l=0}^{N-1} a(l) e^{-j \omega l}}
$$

After applying (4), it was found that the Butterworth IIR filter of order 21 performed similarly to the best filter from the list, the Elliptic IIR filter (Figure 3). In order to improve frequency performance, section $\mathrm{V}$ includes a complete selection study for the Butterworth filters ranging from orders 21 to 39 .

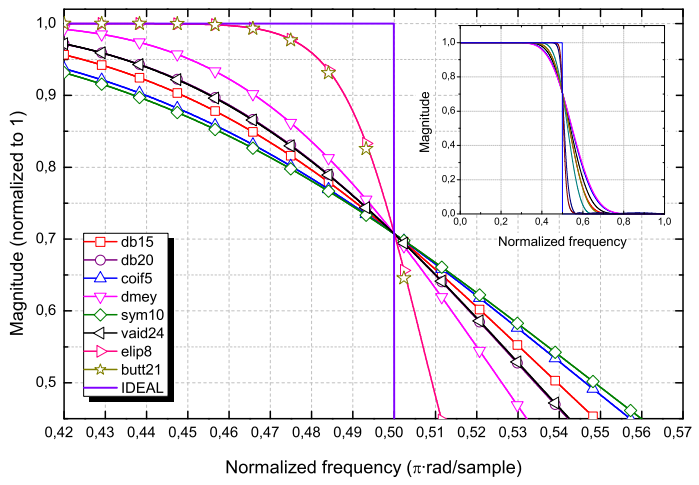

Figure 3: Frequency criterion

To reduce this initial list of filters, a simple frequency criterion has been used. The best decomposition filters have been selected regarding the similarity between their frequency response and the ideal behavior (vertical line in Figure 3). For the sake of clarity, Figure 3 shows only the 8 best 
filters (Butterworth of order 21 overlaps Elliptic's frequency response) regarding their superior frequency discrimination: db15, db20, coif5, dmey, sym10, vaid24, ellip8 and butt21. The cutoff frequency for all these filters corresponds to the frequency value where the gain is $0.707\left(\frac{\sqrt{2}}{2}\right)$ times the highest filter gain value $(-3.01 \mathrm{~dB})$.

From Figure 3, one can clearly see that the two IIR filters, Butterworth and Elliptical, are the best ones in terms of frequency response. Despite their design complexity (output feedback is always necessary), the main advantage of IIR filters relies on their implementation efficiency: with a low number of calculations per time step we get the best frequency discrimination [37]. The use of filter structures with worse frequency characteristics will lead to unexpected and undesirable spectral leakages on every performed convolution. This will result in less energy obtained at the last decomposition level or, in other words, a lower RMS value for the calculated harmonics.

In addition to frequency response, computational cost is another parameter to be considered when selecting the optimal filter for any signal processing application [38]. Figure 4 shows the number of mathematical operations (using the Matlab command cost) that an input sample suffers during the application of each filter. As can be seen, the best structures in order of importance (from less to more number of operations) are: elip8 (IIR), sym10 (FIR), vaid24 (FIR), coif5 (FIR), db15 (FIR), db20 (FIR), but21 (IIR) and finally dmey (FIR). It can be noted that the selection of very long structures would potentially increase the mathematical complexity of the 7levels decomposition tree.

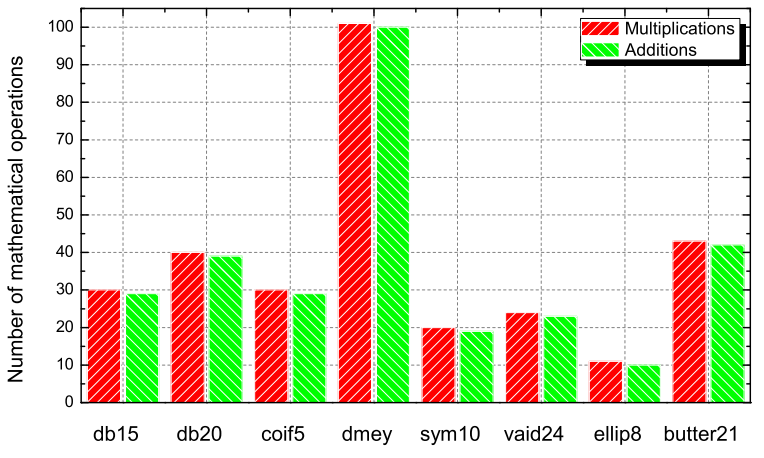

Figure 4: Computational complexity

On the basis of the obtained results, despite its good frequency discrimination, dmey filter was discarded due to its excessive computational cost. Although db15 and sym10 showed a limited number of operations, they were also discarded due to their frequency response far from the ideal behavior. We would like to highlight that the Butterworth filter shows a computational cost that could be considered high, but it is compensated with a good frequency response. To summarize, frequency response and computational cost criteria allowed the selection of filters showing the best balanced performance; these are the Elliptic, Vaidyanathan, Daubechies, Coiflet and Butterworth filters. These structures will be reassessed based on their spectral leakage performance (Section V).

\section{Convolution TeChNiQue SELECTION}

Following the requirements provided in the current IEC standard for harmonic measurements, IEC 61000-4-7 [4], the developed algorithm uses a rectangular shaped window. The most frequent and easiest way to implement the convolution process is to assume infinite signals by extending the waveforms outside the measuring window limits. In this context, circular convolutions may have some artificial edge discontinuities as the number of iterations increases [39]. To overcome these problems at the edges, a symmetric extension can be used but other discontinuities can appear, creating problematic and undesirable effects on the borders [40].

Several types of convolution, depending on the border interpretation, were tested during the development of the method to evaluate their performance [41], [42]: antisymmetric-padding (half-point) (ASYM), antisymmetric padding (whole-point) (ASYMW), symmetric-pading (half-point) (SYM), symmetricpadding (whole-point) (SYMW), smooth-padding of order 1 (SP1) and zero-padding (ZPD). To analyze the best convolution strategy to be adopted, these six 1-D convolution approaches were tested under harmonic evaluation. An ideal $50 \mathrm{~Hz}$ sine waveform with an amplitude of $\sqrt{2}$ p.u. and 10 cycles length (1280 samples at frequency rate of $6400 \mathrm{~S} / \mathrm{s})$ was selected as the test data. The measuring window was extended 1.5 cycles (192 samples) before and beyond the 10 cycles of the initial waveform, giving a total length of $10 \pm 1.5$ cycles (1664 samples). This allowed to extend correctly the waveform depending on the tested strategy (see Figure 5).

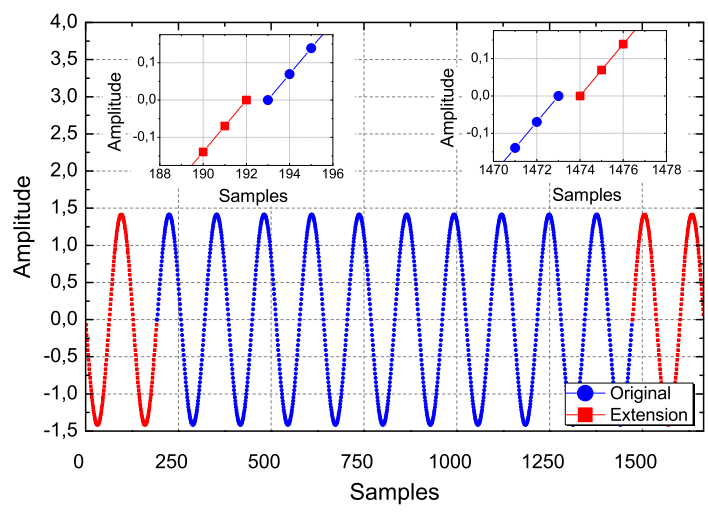

(a) Antisymmetric-padding (half-point)

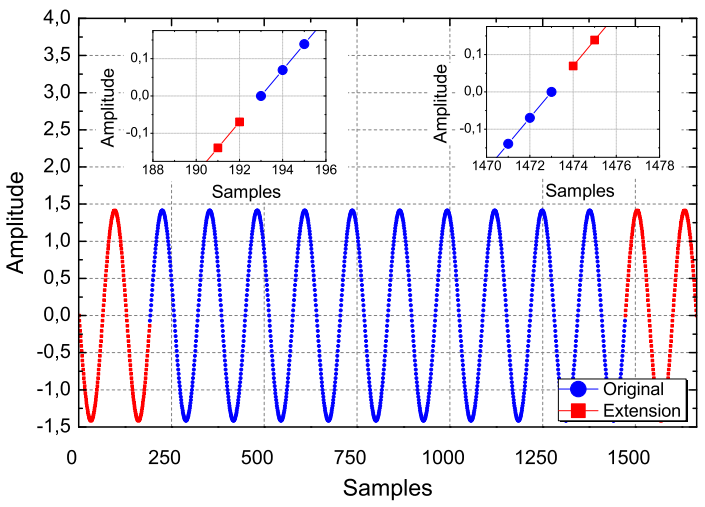

(b) Antisymmetric-padding (whole-point) 


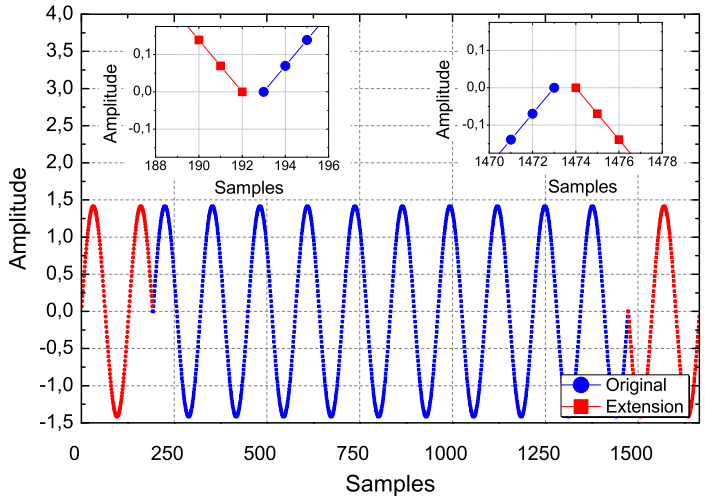

(c) Symmetric-padding (half-point)

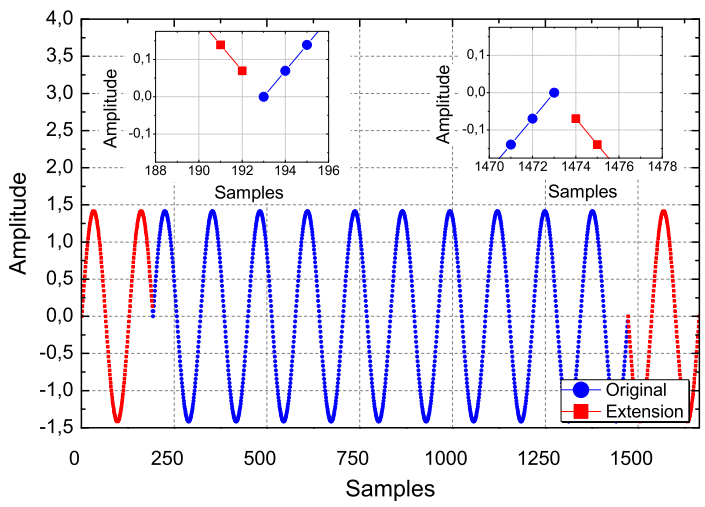

(d) Symmetric-padding (whole-point)

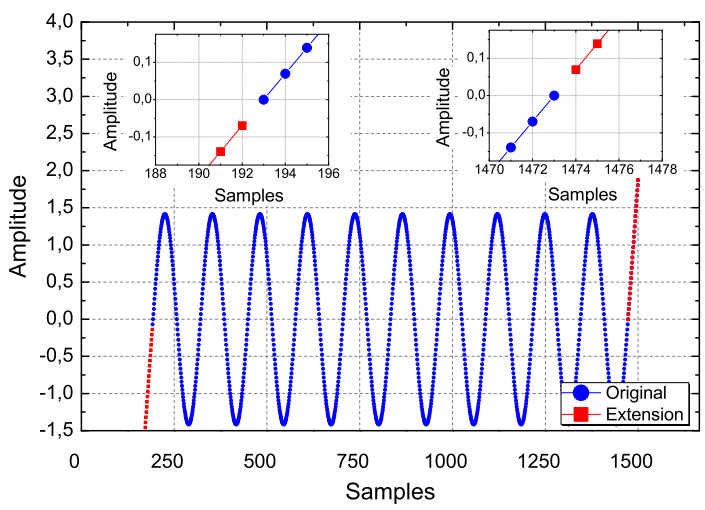

(e) Smooth-padding of order 1

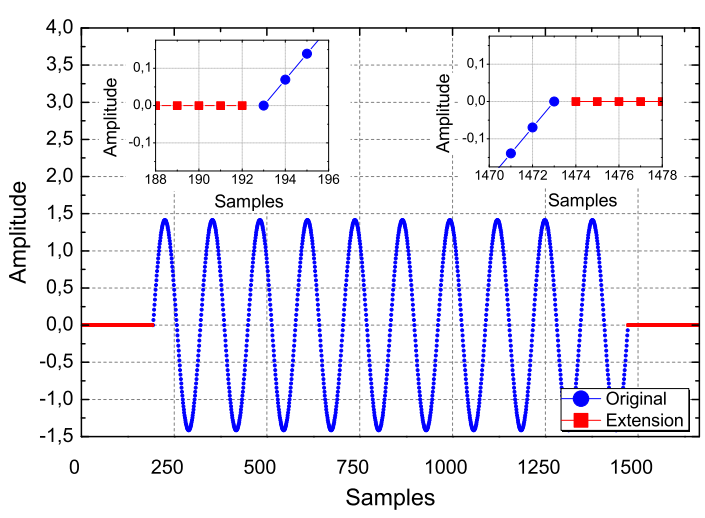

(f) Zero-padding

Figure 5: Circular convolution types
The selection of the best convolution strategy was based on the lowest RMS deviation measured for a pure-tone harmonic waveform. It is assumed that the presented method shall calculate, at least, pure-tone harmonics. Those circular convolution techniques that do not meet this requirement will be automatically discarded. In this regard, it has been implemented the MRA decomposition tree varying the methodology of convolution. The selected core filter for this purpose was the Butterworth IIR of order 21, corresponding to the preliminary best frequency selectivity. Besides, further analysis was carried out in Section V to identify the optimal filter order.

The obtained RMS results after running the presented MRAbased tree are shown in Figure 6.

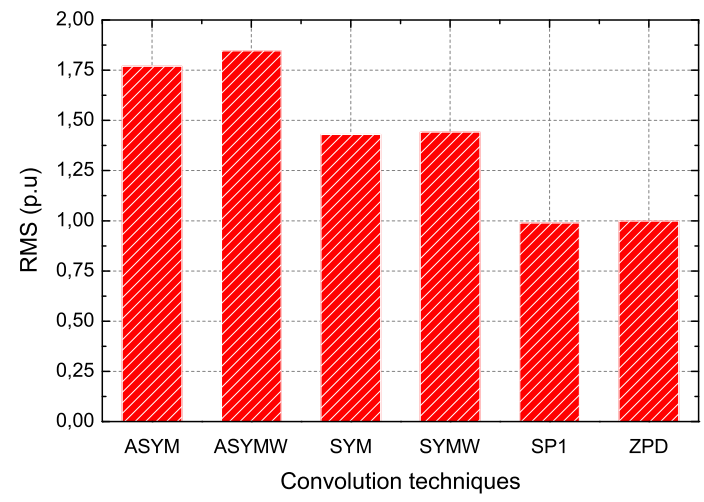

Figure 6: Convolution performance

As can be seen, the smooth padding and the zero padding techniques showed the most accurate RMS evaluation, presenting the last one the smallest undesirable edge effect with the lowest spectral leakage at the last decomposition level $\left(\epsilon_{R M S} \approx 0 \%\right)$. Antisymmetric and symmetric convolution techniques showed important deviations not compatible with a smooth operation of the method. With these premises, the zeropadding method was selected as the most suitable convolution strategy to be implemented on the final MRA decomposition scheme.

\section{HARMONIC TEST RESULTS: SPECTRAL LEAKAGE}

\section{A. Pure-tone harmonics study}

The use of non-ideal filters makes the method imperfect in terms of frequency discrimination. This section includes the analysis of the spectral leakage effect for the first 50 harmonic orders. The testing platform is based on the 7level MRA structure with a 2-node grouping (3) for the harmonic evaluation. Although the system is designed for a real-time harmonic processing, the system performance has been checked through an iterative frequency sweep along the first 50 individual pure-tone harmonics (5).

$$
U_{N}=100 \sqrt{2} \sin (N 2 \pi f t)
$$

where $\omega$ is equal to $100 \pi \mathrm{rad} / \mathrm{s}$ and $N$ is the test index counter from 1 to 50 . 
This methodology provides very valuable information regarding the existing spectral leakage at the last level of the decomposition (RMS evaluation). Figure 7 shows a qualitative logarithmic representation of that spectral leakage effect when the Vaidyanathan-24 (poor), Elliptic-8 (good) and Butterworth21 (excellent) filters are applied.

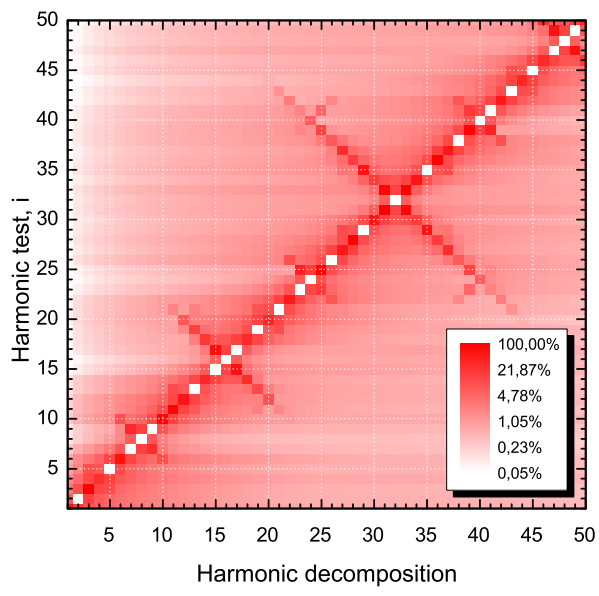

(a) Vaidyanathan filter

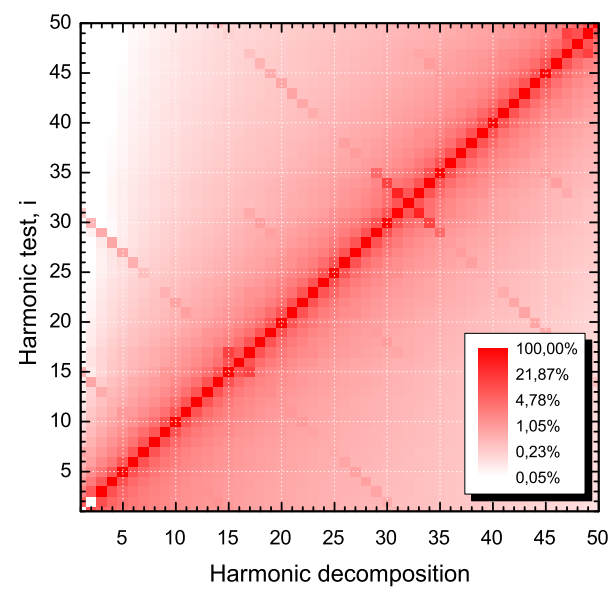

(b) Elliptic filter

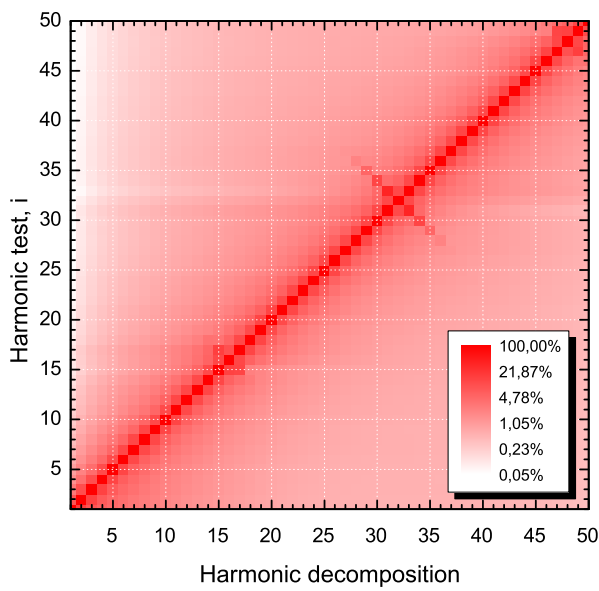

(c) Butterworth filter

Figure 7: Harmonic spectral leakages
As showed in Figure 7a, Figure 7b and Figure 7c, spectral leakages were representative for any given test. For an ideal response, all the energy (100\% RMS values) should be concentrated on the red 45-degree line. As previously mentioned, the non-ideal implementation (filters are not ideal) causes some spectral leakage around the pure tone frequencies, so any content on the surroundings of that ideal red line is related to a certain energy loss. This spurious energy distribution negatively affects the final RMS result, and therefore altering amplitude, phase and frequency readouts.

Table II shows maximum and minimum deviations considering the true RMS as a reference (6), as well as the coefficient of variation $\left(\mathrm{C}_{v}\right)$ that measures the extent of variability in relation to the mean value of the 50 measured pure-tone harmonic (7). Similarly, the Average Energy Leakage (AEL) index is defined in (8) to normalize the mean spectral leakage for the $N$ performed tests (5) across the $M-1$ measured harmonics.

$$
\begin{gathered}
\epsilon_{R M S}(\%)=\left|\frac{R M S_{\text {measured }}-R M S_{\text {true }}}{R M S_{\text {true }}}\right| \cdot 100 \\
C_{v}(\%)=\frac{\sigma}{\mu} \\
A E L(\%)=\frac{\sum_{i=1}^{N}\left(\sqrt{\sum_{\substack{j=1 \\
j \neq i}}^{M} R M S(\%)_{j}^{2}}\right)}{N}
\end{gathered}
$$

Frequency selection criteria (see Section II-A) demonstrated that IIR filters (i.e. Elliptic and Butterworth) were more accurate than FIR filters (i.e. db20, coif5 and vaid24). Additionally, $C_{v}$ parameter showed energy recovery variability, obtaining very precise results when using Elliptic or Butterworth IIR filters in contrast with other FIR filters. Finally, AEL index showed that the spectral leakage effect is important even for IIR filters.

Table II: Pure-tone analysis summary

\begin{tabular}{lcrrrr}
\hline Filter & Coeff. & $\epsilon_{\max }(\%)$ & $\epsilon_{\min }(\%)$ & $C_{v}(\%)$ & AEL (\%) \\
\hline vaid & 24 & 77.300 & 0.004 & 41.534 & 13.899 \\
coif5 & 30 & 24.192 & 0.714 & 7.185 & 18.127 \\
db20 & 40 & 21.981 & 0.666 & 6.070 & 14.552 \\
ellip & 8 & 6.937 & 0.039 & 1.366 & 7.847 \\
butt & 21 & 5.876 & 0.000 & 1.182 & 8.126 \\
\hline
\end{tabular}

It is important to note that the Butteworth filter's roll-off characteristic can be enhanced if the filter order is increased. A complete study was carried out to get the optimal filter order to exceed the performances shown in Table II. Figure 8 shows the results after analyzing the performance of the method using Butterworth IIR filters from order 21 (frequency response similar to Elliptic8) to 39.

Minimum values of AEL index (4.345), $\epsilon_{\max }$ (3.070) and $\epsilon_{\min }(0.017)$ identified the Butterworth's optimal order (29 coefficients) revealing that the spectral leakage effect is minimized. Additionally, computational cost has also been determined. The complete algorithm was run into a $2.26 \mathrm{GHz}$ 


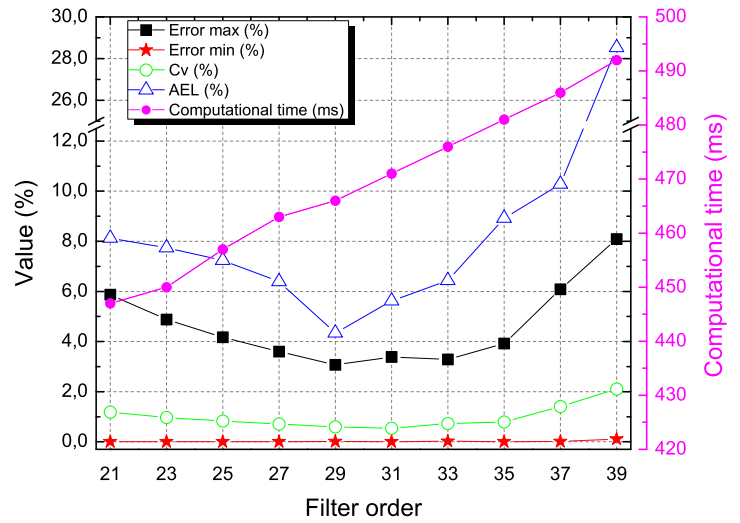

Figure 8: WPD performance vs Butterworth filter order

Inter Core 2 Duo processor with $8 \mathrm{~GB} 1067 \mathrm{MHz}$ DDR3 RAM. Computational time results are referred to only one iteration (one test), enough to identify performance trends. It is clear that as long as the filter order increases, computational effort significantly grows [43]. However, computational costs for all analyzed Butterworth filters were around $475 \pm 25 \mathrm{~ms}$.

In view of the obtained results, it is proved that the optimal filter order corresponded to the $29^{\text {th }}$ reducing by half the deviations obtained by the Elliptic IIR filter. For this reason, this filter will be used on the following subsection for the analysis of more realistic waveforms.

\section{B. Fluctuating harmonics study}

In order to investigate the influence of real fluctuating harmonics on the filter's performance, a set of 50 pure-tone signals with a sharp transition on the amplitude at a certain instant of the measuring window (similar to the one included in the Annex C of the standard [4]) was selected.

$$
U_{N}^{\prime}=100 \sqrt{2} k_{m} \sin (N 2 \pi f t)
$$

where $k_{m}, N, f$ and $t$ are the modulation factor, harmonic order (from 1 to 50), frequency and time respectively.

For this purpose, two different sets of test were built. In Test Set I, the amplitude of the pure-tone signal dropped down to $20 \%$ whereas in Test Set II the same waveform was used with added white Gaussian noise ( $35 \mathrm{~dB})$. These signals simulate real analysis conditions where the amplitude change occurs after $4.054 \cdot h$ cycles of the $h^{\text {th }}$ harmonic order.

$$
\begin{gathered}
\text { (Test Set I) } k_{m}= \begin{cases}1 & \text { if } 0 \mathrm{~ms} \leq t \leq 81.08 \mathrm{~ms}, \\
0.2 & \text { if } 81.08 \mathrm{~ms} \leq t \leq 200 \mathrm{~ms}\end{cases} \\
\text { (Test Set II) } k_{m}= \begin{cases}1+\epsilon & \text { if } 0 \mathrm{~ms} \leq t \leq 81.08 \mathrm{~ms}, \\
0.2+\epsilon & \text { if } 81.08 \mathrm{~ms} \leq t \leq 200 \mathrm{~ms}\end{cases}
\end{gathered}
$$

where $\epsilon$ denotes a $35 \mathrm{~dB}$ additive white Gaussian noise.

A summary of the obtained results after applying the filters is shown in Table III and Table IV. Note that all values were very similar to the ones previously presented in Table
II. Once again, Butterworth IIR filter of order 29 was the most accurate filter according its reported performance indexes $\left(C_{v}\right.$ and AEL) and maximum/minimum deviations from input values $\left(\epsilon_{\max }\right.$ and $\left.\epsilon_{\min }\right)$.

Table III: Test Set I results

\begin{tabular}{lcrrrr}
\hline Filter & Coeff. & $\epsilon_{\max }(\%)$ & $\epsilon_{\min }(\%)$ & $C_{v}(\%)$ & AEL (\%) \\
\hline vaid & 24 & 75.745 & 0.679 & 38.974 & 15.887 \\
coif5 & 30 & 24.782 & 1.444 & 7.158 & 19.495 \\
db20 & 40 & 22.881 & 1.448 & 6.062 & 16.316 \\
ellip & 8 & 7.889 & 0.794 & 1.361 & 10.496 \\
butt & 29 & 3.878 & 0.687 & 0.567 & 5.579 \\
\hline
\end{tabular}

Table IV: Test Set II results

\begin{tabular}{lcrcrc}
\hline Filter & Coeff. & $\epsilon_{\max }(\%)$ & $\epsilon_{\min }(\%)$ & $C_{v}(\%)$ & AEL (\%) \\
\hline vaid & 24 & 75.702 & 0.672 & 38.964 & 15.903 \\
coif5 & 30 & 24.853 & 1.447 & 7.181 & 19.516 \\
db20 & 40 & 22.917 & 1.398 & 6.056 & 16.343 \\
ellip & 8 & 7.938 & 0.760 & 1.356 & 10.522 \\
butt & 29 & 3.916 & 0.642 & 0.589 & 5.593 \\
\hline
\end{tabular}

\section{CONCLUSION}

The aim of this research work was the proposal of an objective methodology for the selection of the most suitable decomposition structure (filter) for harmonic measurements. The suggested method relies on a binary tree providing 7 fulldecomposition levels for the measurement of both even and odd harmonic contents up to $63^{\text {rd }}$ order in contrast with the partial work developed by the previously mentioned authors. First, this paper presents a general approach of the quadraturemirror filters used. Their special characteristics make the binary decomposition tree a very valuable tool ready to be used for harmonic evaluation.

The methodology for calculating harmonic contents at the lowest decomposition level was also reviewed, being necessary to arrange the output information to gather centered RMS values. This methodology is also compliant with the IEC standard requirements [4] for harmonic measurements.

A preliminary list of 51 FIR filters plus 2 IIR filters was tested, including the most representative filters according to relevant scientific papers. Additionally, 6 convolution methods were considered for the implementation of an accurate MRA scheme, becoming clear that the zero-padding 1-D convolution technique showed the best results.

Four performance parameters were defined: $\epsilon_{\max }, \epsilon_{\min }$, $C_{v}$ and AEL for the analysis of data sets of 50 pure-tone input signals. These quantified the spectral leakage across the first 50 harmonic orders. In order to enhance frequency selectivity, a complete study was carried out to select the optimal filter order of the Butterworth IIR filter regarding the previously mentioned indexes and the iteration computational time. It was found that we can increase the order up to the $29^{t h}$ minimizing the undesirable spectral leakage effect on the spectrum. Finally, the analysis of 2 test sets corresponding 
to realistic synthesized fluctuating signals confirmed that the Butterworth IIR filter was the best one compared to the other tested filters from the $1^{\text {st }}$ to the $50^{\text {th }}$ harmonic order.

\section{ACKNOWLEDGMENT}

The authors would like to thank the Spanish National Metrology Institute, Centro Español de Metrología (CEM), for supporting the EURAMET joint research project Power and Energy of which the present paper is a part.

\section{REFERENCES}

[1] H. Ma and A. A. Girgis, "Identification and tracking of harmonic sources in a power system using a Kalman filter," IEEE Transactions on Power Delivery, vol. 11 , no. 3, pp. 1659-1665, 1996.

[2] IEC, "IEC TR 61000-3-7:2008 - Electromagnetic compatibility (EMC) - Part 3-7: Limits - Assessment of emission limits for the connection of fluctuating installations to MV, HV and EHV power systems."

[3] "IEC/TR 61000-3-15 ed1.0, Electromagnetic compatibility (EMC) - Part 3-15: Limits - Assessment of low frequency electromagnetic immunity and emission requirements for dispersed generation systems in LV network," 2011.

[4] IEC, "IEC 61000-4-7 ed2.0 - Electromagnetic compatibility (EMC) - Part 4-7: Testing and measurement techniques - General guide on harmonics and interharmonics measurements and instrumentation, for power supply systems and equipment connected thereto," 2003.

[5] IEEE, "IEEE std 519-2014. IEEE Recommended Practices and Requirements for Harmonic Control in Electrical Power Systems," 2014.

[6] P. Caramia, G. Carpinelli, F. Pezza, and P. Verde, "Power quality degradation effects on PWM voltage source inverter with diode bridge rectifier," Ninth International Conference on Harmonics and Quality of Power. Proceedings (Cat. No.00EX441), vol. 2, 2000.

[7] P. Wright, "A method for the calibration of harmonic analysers using signals containing fluctuating harmonics in support of IEC61000-3-2," IEE Proceedings - Science, Measurement and Technology, vol. 152, no. 3, pp. 103-109, 2005.

[8] X. Yan, S. Tan, J. Wang, and Y. Wang, "A high accuracy harmonic analysis method based on all-phase and interpolated FFT in power system," Asia-Pacific Power and Energy Engineering Conference, APPEEC, pp. $1-4,2011$.

[9] M. Stéphane, A Wavelet Tour of Signal Processing. Elsevier, 2009.

[10] M. H. J. Bollen, Signal Processing of Power Quality Disturbances. IEEE Press Series on Power Engineering, 2006.

[11] J. Barros, R. I. Diego, and M. de Apraiz, "Applications of wavelet transforms in electric power quality: Harmonic distortion," IEEE International Workshop on Applied Measurements for Power Systems (AMPS), pp. 13-18, 2011.

[12] M. De Apráiz, J. Barros, and R. I. Diego, "A real-time method for timefrequency detection of transient disturbances in voltage supply systems," Electric Power Systems Research, vol. 108, pp. 103-112, 2014.

[13] M. B. Latran and A. Teke, "A novel wavelet transform based voltage sag/swell detection algorithm," International Journal of Electrical Power \& Energy Systems, vol. 71, pp. 131-139, 2015.

[14] S. Santoso, W. M. Grady, S. Member, E. J. E. Powers, J. Lamoree, and S. C. S. Bhatt, "Characterization of distribution power quality events with Fourier and wavelet transforms," IEEE Transactions on Power Delivery, vol. 15, no. 1, pp. 247-254, 2000

[15] W. G. Morsi and M. E. El-Hawary, "On the application of wavelet transform for symmetrical components computations in the presence of stationary and non-stationary power quality disturbances," Electric Power Systems Research, vol. 81, no. 7, pp. 1373-1380, 2011.

[16] S. Avdakovic, A. Nuhanovic, M. Kusljugic, and M. Music, "Wavelet transform applications in power system dynamics," Electric Power Systems Research, vol. 83, no. 1, pp. 237-245, 2012.

[17] W. G. Morsi, "Electronic reactive energy meters' performance evaluation in environment contaminated with power quality disturbances," Electric Power Systems Research, vol. 84, no. 1, pp. 201-205, 2012.

[18] S. Deokar and L. Waghmare, "Integrated DWT-FFT approach for detection and classification of power quality disturbances," International Journal of Electrical Power \& Energy Systems, vol. 61, pp. 594-605, 2014.

[19] H. M. Al-Hamadi, "Fuzzy logic voltage flicker estimation using Kalman filter," International Journal of Electrical Power \& Energy Systems, vol. 36 , no. 1 , pp. $60-67,2012$.
[20] V. Apetrei, C. Filote, and A. Graur, "Harmonic analysis based on Discrete Wavelet Transform in electric power systems," Ninth International Conference on Ecological Vehicles and Renewable Energies (EVER), pp. $1-8,2014$.

[21] X. Jiao, K. Ding, and G. He, "An algorithm for improving the coefficient accuracy of wavelet packet analysis," Measurement: Journal of the International Measurement Confederation, vol. 47, no. 1, pp. 207-220, 2014.

[22] C. A. Duque, P. M. Silveira, and P. F. Ribeiro, "Visualizing time-varying harmonics using filter banks," Electric Power Systems Research, vol. 81, no. 4, pp. 974-983, 2011

[23] C. A. Naik and P. Kundu, "Power quality index based on discrete wavelet transform," International Journal of Electrical Power \& Energy Systems, vol. 53, pp. 994-1002, 2013.

[24] S.-X. Zhu and H. Liu, "Simulation study of Power Harmonic Based on Daubechies Wavelet," IEEE International Conference on e-product, e-service and e-entertainment (ICEEE), pp. 1-4, 2010.

[25] C. Yuan, X. Li, X. Zhao, and W. Wang, "Research of the Harmonic Detection Method Based on Wavelet Transform," IEEE International Conference on Control, Automation and Systems Engineering (CASE), pp. 1-4, 2011.

[26] L. Eren, M. Unal, and M. Devaney, "Harmonic Analysis Via Wavelet Packet Decomposition Using Special Elliptic Half-Band Filters," IEEE Transactions on Instrumentation and Measurement, vol. 56, no. 6, pp. 2289-2293, 2007.

[27] B. Kotnik and Z. Kačič, "A noise robust feature extraction algorithm using joint wavelet packet subband decomposition and AR modeling of speech signals," Signal Processing, vol. 87, no. 6, pp. 1202-1223, 2007.

[28] J.-y. Chen and Y. Hai, "RMS and Power Measurements based on Wavelet Packet Decomposition using Special IIR Filter Bank," International Conference on Electronic and Mechanical Engineering and Information Technology (EMEIT), vol. 1, pp. 1-4, 2011.

[29] D. K. Alves, C. M. S. Neto, F. B. Costa, and R. L. A. Ribeiro, "Power Measurement Using the Maximal Overlap Discrete Wavelet Transform," 11th IEEE/IAS International Conference on Industry Applications (INDUSCON), no. 1, pp. 1-7, 2014

[30] M. Markovska and D. Taskovski, "Polyphase representation of QMF filter bank for power systems harmonics analysis," IEEE International Conference on Computer as a Tool (EUROCON), pp. 1-6, 2015.

[31] L. C. M. D. Andrade, R. Augusto, and S. Fernandes, "Adaptive Threshold for Segmentation of Combined Power Quality Disturbances," PowerTech, IEEE Eindhoven, pp. 1-5, 2015.

[32] K. Gröchenig, Foundations of Time-Frequency Analysis. Birkhauser, 2001.

[33] J. Barros, R. I. Diego, and M. Apraiz, "Applications of wavelet transform for analysis of harmonic distortion in power systems: A review," IEEE Transactions on Instrumentation and Measurement, vol. 61, no. 10, pp. 2604-2611, 2012.

[34] A. Bogges and F. J. Narcowich, A First Course in Wavelets with Fourier Analysis. Prentice Hall Upper Saddle River, NJ, 2001.

[35] B. B. Hubbard, The world according to wavelets: the story of a mathematical technique in the making. A. K. Peters, Ltd., 1996.

[36] W.-K. Yoon and M. J. Devaney, "Reactive power measurement using the wavelet transform," IEEE Transactions on Instrumentation and Measurement, vol. 49, no. 5, pp. 1205-1210, 2000.

[37] S. Winder, Analog and Digital Filter Design. Elsevier, 2002.

[38] A. H. Ghaemi, H. Askarian Abyaneh, and K. Mazlumi, "Harmonic indices assessment by Wavelet Transform," International Journal of Electrical Power and Energy Systems, vol. 33, no. 8, pp. 1399-1409, 2011.

[39] B.-C. Wang, Digital Signal Processing Techniques and Applications in Radar Image Processing. New York, NY, USA: Wiley - Interscience, 2008.

[40] S. A. Martucci, "Symmetric convolution and the discrete sine and cosine transforms," IEEE Transactions on Signal Processing, vol. 42, no. 5, pp. 1038-1051, 1994

[41] I. Ito and H. Kiya, "A computing method for linear convolution in the DCT domain," European Signal Processing Conference, no. Eusipco, pp. 323-327, 2011.

[42] E. R. Pacola, V. I. Quandt, F. K. Schneider, and M. A. Sovierzoski, "The Wavelet Transform Border Effect in EEG Spike Signals," World Congress on Medical Physics and Biomedical Engineering May 26-31, 2012, Beijing, China SE - 155, vol. 39, pp. 593-596, 2013.

[43] W. G. Morsi, "A wavelet-based approach for reactive power metering in modern three-phase grids considering time-varying power quality disturbances," Electric Power Systems Research, vol. 87, pp. 31-38, 2012 . 


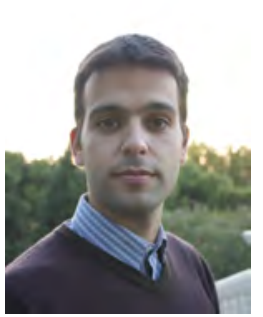

Jorge Bruna. He was born in Zaragoza, Spain, in 1983. He received his M.Sc. degree in Renewable Energies and Energy Efficiency and his Ph.D. degree in Electric Engineering from the University of Zaragoza (Spain) in 2011 and 2016, respectively.

He is currently leading the CIRCE Electrical Tests and Studies Group since 2012. He is working at the LME-CIRCE laboratory as a researcher since 2008. Additionally, he has published several scientific papers in the field of Power Quality disturbances and also developed some advanced measurements algorithms for monitoring Power Quality parameters.

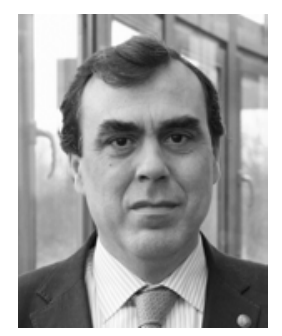

Julio J. Melero. He received his Ph.D. in Physics from the University of Zaragoza, Spain, in 1997. $\mathrm{He}$ is currently working as senior lecturer with the Electrical Engineering Department of the University of Zaragoza. He collaborates with CIRCE Foundation since 2001, leading a group of 30 researchers in Power Quality, Electrical Metrology and Wind Energy.

He has published more than 10 scientific papers and participated in around 30 congresses. Currently, he is the Field Tests Technical Responsible of the Metrology Laboratory LME-CIRCE, accredited for Low Voltage distribution Power Networks. His current research topics are Power Quality, Electrical Metrology and Wind Energy. 